\title{
Reliability of a new test battery for fitness assessment of the European Astronaut corps
}

\author{
Nora Petersen 1,2,5* ${ }^{*}$ Lutz Thieschäfer ${ }^{3}$, Lori Ploutz-Snyder ${ }^{4}$, Volker Damann² and Joachim Mester ${ }^{5}$
}

\begin{abstract}
Background: To optimise health for space missions, European astronauts follow specific conditioning programs before, during and after their flights. To evaluate the effectiveness of these programs, the European Space Agency conducts an Astronaut Fitness Assessment (AFA), but the test-retest reliability of elements within it remains unexamined. The reliability study described here presents a scientific basis for implementing the AFA, but also highlights challenges faced by operational teams supporting humans in such unique environments, especially with respect to health and fitness monitoring of crew members travelling not only into space, but also across the world. The AFA tests assessed parameters known to be affected by prolonged exposure to microgravity: aerobic capacity $\left(V^{2} \mathrm{O}_{2 \mathrm{x}}\right)$, muscular strength (one repetition max, 1 RM) and power (vertical jumps), core stability, flexibility and balance. Intraclass correlation coefficients (ICC ${ }_{3.1}$ ), standard error of measurement and coefficient of variation were used to assess relative and absolute test-retest reliability.

Results: Squat and bench 1 RM $\left(I C C_{3.1}=0.94-0.99\right)$, hip flexion $\left(I C C_{3.1}=0.99\right)$ and left and right handgrip strength $\left(I C C_{3.1}=0.95\right.$ and 0.97$)$, showed the highest test-retest reliability, followed by $V_{2 \max }\left(I C C_{3.1}=0.91\right)$, core strength $\left(I C C_{3.1}=0.78-0.89\right)$, hip extension $\left(I C C_{3.1}=0.63\right)$, the countermeasure $\left(I C C_{3.1}=0.76\right)$ and squat $\left(I C C_{3.1}=0.63\right)$ jumps, and single right- and left-leg jump height $\left(\mathrm{ICC}_{3.1}=0.51\right.$ and 0.14$)$. For balance, relative reliability ranged from $I C C_{3.1}=0.78$ for path length (two legs, head tilted back, eyes open) to $\mathrm{ICC}_{3.1}=0.04$ for average rotation velocity (one leg, eyes closed).

Conclusions: In a small sample $(n=8)$ of young, healthy individuals, the AFA battery of tests demonstrated acceptable test-retest reliability for most parameters except some balance and single-leg jump tasks. These findings suggest that, for the application with astronauts, most AFA tests appear appropriate to be maintained in the test battery, but that some elements may be unreliable, and require either modification (duration, selection of task) or removal (singleleg jump, balance test on sphere) from the battery. The test battery is mobile and universally applicable for occupational and general fitness assessment by its comprehensive composition of tests covering many systems involved in whole body movement.
\end{abstract}

Keywords: Astronaut fitness assessment, Space flight, Test battery, Reliability, Intraclass correlation coefficients (ICC)

\footnotetext{
*Correspondence: Nora.Petersen@esa.int

2 Space Medicine Office (HSO-AM), European Astronaut Centre

Department (ESA), Directorate of Human Spaceflight and Operations

(D/HSO), European Space Agency, Geb. 12, Linder Höhe, PO Box 906096,

51147 Cologne, Germany

Full list of author information is available at the end of the article
} 


\section{Background}

Assessments of physical fitness are frequently used in occupational health care settings to determine an individual's suitability to operate in a specific environment and their capacity to perform required occupational activities without risk to their health and safety, or that of their colleagues [1,2].

When performed periodically and systematically, these assessments may help identify small changes in an individual's physical condition that could compromise their performance and/or ability to work safely, which can then be addressed with remedial action. Physical fitness assessments with European Space Agency (ESA) astronauts are performed to objectively quantify physical performance changes after return from space flight. To increase the quality of the data produced and support both operational and research needs, the former simple, gym-based testing protocol was replaced by the ESA's European Astronaut Centre (EAC) Astronaut Fitness Assessment (AFA), a broader, instrumented test battery. An additional consideration is that the AFA setup must be mobile, as ESA astronauts returning from the International Space Station (ISS) may need to be tested away from ESA facilities, both in the United States and Russia, where post-flight rehabilitation is sometimes implemented. As such, not only must the test elements assess systems affected by space flight and comply with sport scientific evaluation standards, but the test equipment must also be portable and the assessment procedures implementable in various gym environments. This requires a simple test setup, but one that is still capable of producing meaningful data under "field testing" conditions, rather than the standard laboratory conditions available at EAC.

Ten individual tests are included in the AFA. These consider astronauts' unique occupational performance profile, which is characterised by specific tasks and environmental factors, such as launch and landing, extravehicular activities (space 'walks') and ISS-specific operations whilst being exposed to microgravity $(\mu \mathrm{G})$, and ultimately the return into the Earth's gravity. Microgravity exposure for up to 6 months is known to induce decreases in muscle strength $[3,4]$, bone mineral density $[5,6]$, cardiovascular endurance $[7,8]$ and postural control [9-12], and the AFA tests are included based on this current knowledge: anthropometry (height, body mass, and body composition), hip flexibility, handgrip strength, balance, posture and gait characteristics, core, lower and upper body muscle strength, vertical jump (muscular power) and cardiovascular capacity.

A further difficulty in the development of the AFA test battery is the lack of a precise definition of the physical occupational demands of spaceflight. However, although test validity in relation to space flight occupational performance cannot be assessed at this time, the reliability of the new test battery can and should be assessed. This has not been performed previously, because EAC's remit is to provide operational support to ESA astronauts and, historically, it has not had the resources to perform research activities. In addition, the test battery was established for organizational reasons (i.e. an increasing number of ESA long-duration space missions and increased independence of ESA from the other ISS Partners) and the battery was developed and used in parallel to operational implementation, and has undergone numerous changes in the course of development.

Towards this end, the purpose of this investigation was to report the reliability (retest correlation, systematic bias and random error) of each test element, to support the decision to keep, modify or remove them from the AFA.

\section{Methods}

\section{Participants}

Ten male subjects were recruited to participate in the study. The inclusion criteria were based on anthropometric selection standards for ESA Astronauts: healthy and matching the astronaut population in terms of body height (between 149.5 and $190.5 \mathrm{~cm}$ ) and body mass $(\leq 95.0 \mathrm{~kg})$ [13]. The study was approved by the ethical board of the German Sport University in Cologne and all subjects provided informed written consent before participation.

\section{Study design}

The study used a test-retest design in a controlled laboratory environment, with participants making three visits to the German Sport University, with each visit separated by 7 days. Prior to the first experimental visit, participants performed a familiarisation session of the entire test battery. For experimental visits, participants arrived at approximately the same time of day, wearing the same clothes and shoes for each visit. They were instructed to not deviate from their usual training and eating habits during the testing period. To minimise measurement errors, subject position, movement speed, observer instructions, measuring instrument, location and test conditions were standardised between sessions.

The test elements were always implemented in the same order, with the aim of minimising fatigue effects (e.g. elements with a low physical demand were scheduled at the beginning of the battery prior to implementation and those requiring significant/maximal physical effort at the end) with 1-3-min rest breaks between measurements and, as with the AFA performed with ESA astronauts, all elements were completed in a 2-h time period. Consistent with normal AFA procedures, subjects ran 
on a treadmill for $10 \mathrm{~min}$ at $10 \mathrm{~km} \mathrm{~h}^{-1}$ to warm up and no other specific warm-up exercises were completed. To avoid observer bias, all experimental staff were familiarised with the tests to which they were assigned and they conducted these tests for the entire study.

\section{Anthropometry}

Height was measured using a stadiometer (SECA GmbH, Hamburg, Germany). Body mass was measured and percentage body fat estimated using a combined weighing scale and bio-electrical impedance device (BC-418 MA, Tanita, Tokyo, Japan).

\section{Flexibility}

Hip flexion was measured with a Sit-and-Reach box (Sport Time, USA). Participants were instructed to reach forward as far as possible in a slow and controlled movement and hold the final position for $2 \mathrm{~s}$. The distance (in $\mathrm{cm}$ ) achieved was measured and three trials were performed, with the single best effort used for analysis.

Hip extension was measured using a modified Thomas Test [14]. Participants adopted a supine position on a bench with both legs bent over the edge. Allowing the measured leg to hang freely, participants were instructed to pull the other knee to their chest ensuring continuous firm contact of the lumbar spine with the bench surface. Hip angle $\left(^{\circ}\right)$ in relation to the bench surface was measured using an inclinometer (ACU 360, Lafayette Instrument Company, Lafayette, USA) at the mid-thigh, capturing six consecutive values in the same position. The average of those six values was used for analysis. An identical measurement was then made with the other leg.

\section{Handgrip strength}

Maximal, one-handed handgrip strength was measured for both hands using a mechanical handgrip dynamometer (Takei Scientific Instruments Co. Ltd., Niigata City, Japan). From a standing position, with their arm down by their side, participants were instructed to apply maximal force for $2 \mathrm{~s}$. Participants made three attempts per hand, alternating hands each time, separated by at least $60 \mathrm{~s}$ rest, with the single best effort used for analysis.

\section{Core strength}

The ability to maintain a standardised position and movement was measured in three different (ventral, lateral and dorsal-in that order) positions as described in the Swiss Olympic manual of core strength assessment [15]. In each position, participants were requested to maintain both position and speed of movement $(1 \mathrm{~Hz})$ in synchronisation with a metronome (Ma-30, KORG metronome, Tokyo, Japan). The test was terminated when the subject was unable to maintain the required position or movement [15] after either a maximum of two warnings by the test observer or until volitional fatigue. The time (in s) to test termination was recorded in all three positions.

\section{Muscle strength}

Muscle strength was assessed by estimating the one repetition maximum (1 RM) using the Brzycki Formula $[16,17]$. Bench press and squat manoeuvres were conducted in a standardised body position (feet, hands, and bench) and range of motion in relation to the rack (Smith machine, gym80, International $\mathrm{GmbH}$, Gelsenkirchen, Germany). Participants were instructed to perform as many repetitions as possible at a pre-selected load with the aim of achieving volitional fatigue in less than 10 repetitions.

\section{Balance}

Ten tests of balance were performed using two different instruments (Table 1). To assess postural sway area of the body's centre of pressure (COP) and COP displacement path length, six tests (Levels 1-6), each with an increasing level of difficulty, were performed on a pressure distribution platform (FDM-S Pressure Plate, Zebris Medical GmbH, Isny, Germany). Data were processed at $100 \mathrm{~Hz}$ using Zebris software, with COP area taken as the area (in $\mathrm{mm}$ ) within the $95 \%$ confidence interval. The last four tests (Levels 7-10) were performed on a balance board (Fig. 1) with a metal spherical base (Sport Thieme GmbH, Grasleben, Germany) instrumented with an inclinometer (BalensoSenso, Fa. Reinert, Pforzheim, Germany) inserted into the sphere underneath the board to measure angular velocity.

Test conditions with both devices were made increasingly difficult by closing the eyes, standing on one foot, tilting the head back and standing on tiptoes, which was a

Table 1 Balance test Levels 1-10, implemented on pressure plate and balance board

\begin{tabular}{ll}
\hline Difficulty level & Test conditions \\
\hline Pressure plate & Tasks \\
Level 1 & Both feet, eyes closed \\
Level 2 & One foot, eyes open \\
Level 3 & One foot, eyes closed \\
Level 4 & Both foot, eyes open, head tilt \\
Level 5 & Both feet, tip toes, eyes open \\
Level 6 & Both feet, tip toes, eyes closed \\
Balance board & \\
Level 7 & Both feet, eyes open \\
Level 8 & One foot, eyes open \\
Level 9 & Both feet, eyes closed \\
Level 10 & One foot, eyes closed \\
\hline
\end{tabular}




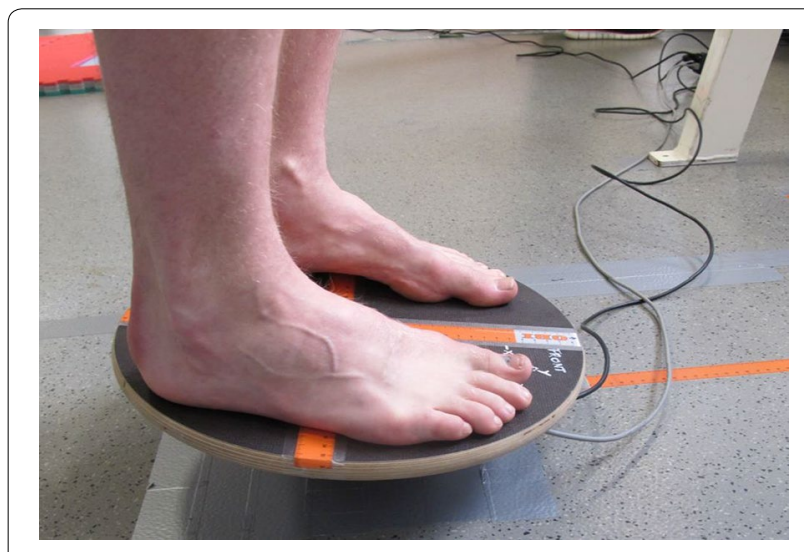

Fig. 1 Instrumented balance board used for balance tasks (L7-10).

modification of existing balance tests in rehabilitation practice [18]. Foot and hand positions were standardised (hands on hips, surface markers for feet) and tests on one foot were always performed with the same leg. All tests lasted a maximum of $15 \mathrm{~s}$. Stepping off the device surface, the hands losing contact with the hips (e.g. to grasp safety handles) and opening the eyes (for tests with eyes closed) were termination criteria for any test. In the case of termination, the maximum time achieved by the subject was recorded. All ten tests were completed in the same order, regardless of the subject's ability to complete the full $15 \mathrm{~s}$ for any test.

\section{Muscle power}

Lower body muscle power was assessed from a countermovement jump (CMJ), squat jump (SJ), single-leg CMJ jumps [right (SLJ-R) and left (SLJ-L) leg] and a drop jump
(DJ) from a $0.28-\mathrm{m}$ platform. In bare feet, participants were instructed to jump as high as possible whilst keeping their hands in contact with their hips at all times. Each jump was attempted three times, with a break of $60 \mathrm{~s}$ between jumps and the single best effort for each task was used for analysis. Performance was measured by calculating jump height $(\mathrm{m})$ based on measurement of GRF (N), contact time (s) and rate of force development (RFD) (N/s) were and using a force platform (5691 A, Kistler, Winterthur, Switzerland) and analysis software (TEMPLO@ by Contemplas GmbH, Kempten, Germany) with a sampling rate of $300 \mathrm{~Hz}$ [19]. Reactive strength index (RSI) was also calculated for the drop jump as a measure of stretch-shortening cycle function.

\section{Aerobic capacity}

Aerobic capacity $\left(V \mathrm{O}_{2 \max }\right)$ was measured on a treadmill (PPS 55med-I, WOODWAY GmbH., Weil am Rhein, Germany) using a modified Bruce protocol [20] (Fig. 2). Belt speed was increased by $1.8 \mathrm{~km} \mathrm{~h}^{-1}$ every $3 \mathrm{~min}$ (starting at $6 \mathrm{~km} \cdot \mathrm{h}^{-1}$ ) at a constant $1 \%$ incline until volitional fatigue, with 30-s breaks between intervals for lactate sampling ("Lactate scout", EKF-diagnostic GmbH, Magdeburg, Germany).

Oxygen uptake was measured continuously using a spirometry system (Zan600, ZAN Austria e.U., SteyrDietach, Austria) and $V \mathrm{O}_{2 \max }$ calculated from a sliding mean over the last $30 \mathrm{~s}$ before fatigue. Heart rate was recorded using a chest strap and watch (RS800, POLAR, Kempele, Finland). Earlobe lactate samples were taken 1 and $5 \mathrm{~min}$ after the point of fatigue, and all lactate values inserted into the ERGONIZER ${ }^{\circledR}$ software (ERGONIZER ${ }^{\circledR}$

(modf.) Bruce protocol

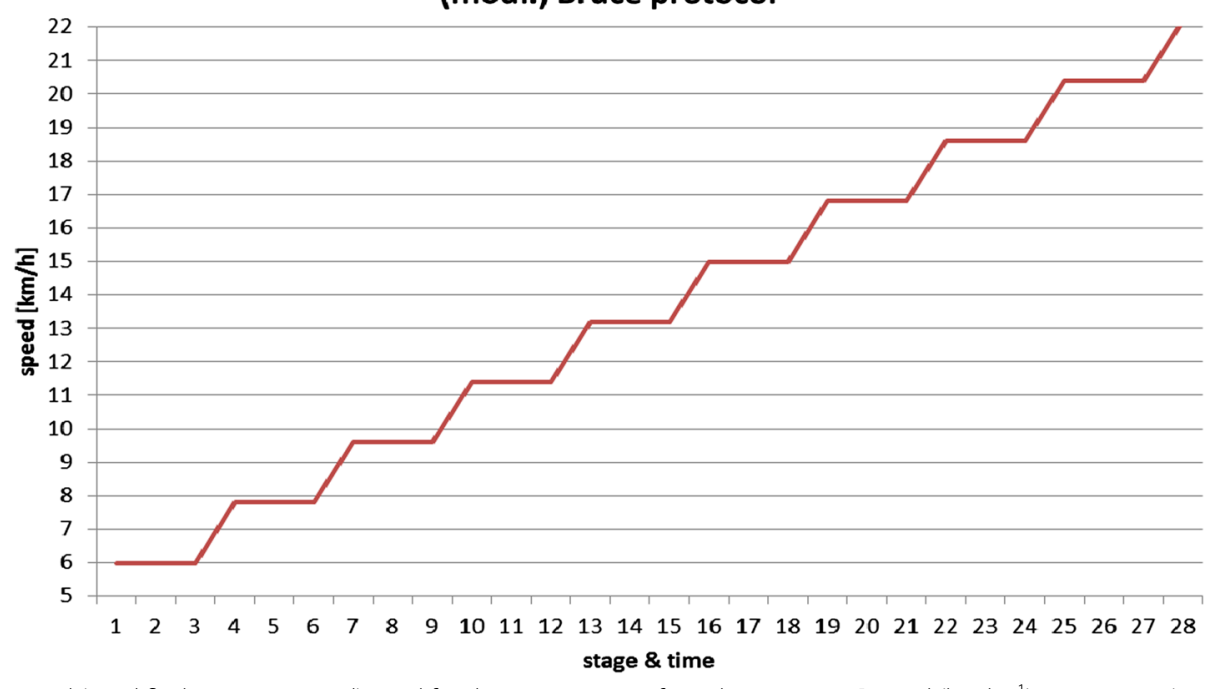

Fig. 2 Treadmill protocol (modified Bruce protocol) used for the assessment of aerobic capacity [speed (km $\mathrm{h}^{-1}$ ); stage time (min)]. 
version 4.1.10, Kai Röcker, Freiburg, Germany) to provide a secondary estimation of $V \mathrm{O}_{2 \max }$. In the AFA performed with astronauts, this estimation technique is used when the spirometry equipment is not available to make a direct measurement.

\section{Statistical analysis}

Data are reported as mean \pm 1 SD unless otherwise stated. The main objectives of the assessment were to evaluate relative (Intraclass correlation coefficients, $\mathrm{ICC}_{3.1}$ ) with fixed raters, and absolute (standard error of measurement, SEM, and coefficient of variation, CV) reliability of each element. The rationale for the fixed raters was that, in the operational implementation of the AFA, an individual astronaut is always tested by the same person for consecutive AFAs, and thus, in this study, the same operators always conducted specific test elements and inter-rater correlations were not assessed.

Data from the three experimental visits were analysed using a repeated measures analysis of variance (ANOVA) $(k=3 ; \alpha$-level $=0.05)$ to calculate SEM, $\mathrm{ICC}_{3.1}$, and the $F$-ratio, to identify systematic bias (critical $F$ value $>3.74$ ) potentially caused by implementation and analysis procedures, learning and/or fatigue effects [21-23]. Prior to analysis, data were tested for normal distribution and homoscedasticity, and, where not evident, a transformation was applied. Thus, a log100 transformation was applied to the following data: balance: COP sway area (Level 1, 2, 3, 5 and 6), path length (Level 4 and 5), and average rotation velocity (Level 7 and 8); jump: CMJ (height and RFD), SJ (height and RFD), SLJ (RFD for both legs jump height for SLJ-L) and DJ (RFD); $V O_{2 m a x}$ : estimation by ERGONIZER ${ }^{\circledR}$; core strength: dorsal position. The measures of error (SEM, CV) are reported in absolute form (' $+/-$ '), or in ratio form (' $\times / \div$ ') for log100 transformed data. Normal distribution or homogeneity, although statistically tested here, may still differ for a larger sample and, therefore, both SEM and CV are always provided. Statistical analysis was performed with commercially available software (PASW Statistics 18, IBM Corporation, Armonk, USA) and "Microsoft Excel 2013" (Microsoft, Redmond, USA).

\section{Results}

Of the ten participants who were recruited into the study, only eight [(mean \pm 1 SD) age $25 \pm 2$ years; height $1.78 \pm 0.05 \mathrm{~m}$; body mass $76.6 \pm 8.6 \mathrm{~kg}]$ completed all the required procedures and were thus included in the statistical analysis.

\section{Anthropometry}

Relative reliability for anthropometric parameters were: body mass: $\mathrm{ICC}_{3.1}=0.99 ; \mathrm{SEM}=0.73 \mathrm{~kg}$; height: $\mathrm{ICC}_{3.1}=0.99 ; \mathrm{SEM}=0.23 \mathrm{~cm}$ and \% body fat $\mathrm{ICC}_{3.1}=0.89 ; \mathrm{SEM}=1.80 \%$ (Table 2). No systematic error was detected with the $F$ test.

\section{Flexibility}

Hip flexion (Sit-and-Reach test) showed a correlation of $\mathrm{ICC}_{3.1}=0.99$; SEM $=1.20 \mathrm{~cm}$, with hip extension (Thomas Test) showing a correlation of $\mathrm{ICC}_{3.1}=0.85$; $\mathrm{SEM}=2.69^{\circ}$ (right leg) and $\mathrm{ICC}_{3.1}=0.63 ; \mathrm{SEM}=4.12^{\circ}$ (left leg) (Table 2).

\section{Muscle strength}

Handgrip strength (right and left hand) showed correlations of $\mathrm{ICC}_{3.1}=0.97, \mathrm{SEM}=1.96 \mathrm{~kg}$ and $\mathrm{ICC}_{3.1}=0.95$; $\mathrm{SEM}=2.50 \mathrm{~kg}$, but also revealed a systematic error in the $F$ test $(F=7.10, P=0.01)$ for the right hand only across all trials.

Time to termination in the core strength tests showed correlations of $\mathrm{ICC}_{3.1}=0.89 ; \mathrm{SEM}=12.66 \mathrm{~s}$ and $\mathrm{ICC}_{3.1}=0.86 ; \mathrm{SEM}=7.90 \mathrm{~s}$ for the ventral and lateral positions. Data from dorsal position demonstrated a lower correlation of $\mathrm{ICC}_{3.1}=0.78$; SEM $=1.11 \mathrm{~s}$ (Table 3; Fig. 3).

The 1 RM strength estimate tests showed correlations of $\mathrm{ICC}_{3.1}=0.99 ; \mathrm{SEM}=2.48 \mathrm{~kg}$ for bench press and $\mathrm{ICC}_{3.1}=0.94 ; \mathrm{SEM}=8.67 \mathrm{~kg}$ for squat (Table 3 ). A systematic error was identified with the $F$ test $[6.18$ $(P=0.01)]$ for squat.

\section{Balance}

Relative reliability ranged from $\mathrm{ICC}_{3.1}=0.80$ for path length on Level 4 on the pressure plate (both legs, head tilted back, eyes open) to $\mathrm{ICC}_{3.1}=0.04$ for average rotation velocity in Level 9 on the balance board (Table 4). Measurement precision indicated error ranges between $18.2 \%$ (CV of COP path length Level 2) and $89.7 \%$ (CV of COP sway area Level 6).

Table 2 Relative and absolute reliability of anthropometry and flexibility measures

\begin{tabular}{lclll}
\hline Parameter & $\begin{array}{l}\text { Pooled } \\
\text { mean } \pm \text { SD }\end{array}$ & $\begin{array}{l}\text { Relative } \\
\text { reliability }\end{array}$ & \multicolumn{2}{l}{$\begin{array}{l}\text { Absolute } \\
\text { reliability }\end{array}$} \\
\cline { 4 - 6 } & & ICC $_{3.1}$ & SEM & CV \\
\hline Body mass (kg) & $76.8 \pm 9.7$ & 0.99 & \pm 0.73 & \pm 0.95 \\
Height $(\mathrm{cm})$ & $180.0 \pm 5.2$ & 0.99 & \pm 0.23 & \pm 0.13 \\
Body fat (\%) & $11.4 \pm 5.3$ & 0.89 & \pm 1.80 & \pm 15.81 \\
Sit-and-Reach (cm) & $27.3 \pm 9.8$ & 0.99 & \pm 1.20 & \pm 4.41 \\
Thomas Test right $\left(^{\circ}\right)$ & $23.1 \pm 7.0$ & 0.85 & \pm 2.69 & \pm 11.63 \\
Thomas Test left $\left(^{\circ}\right)$ & $24.8 \pm 6.7$ & 0.63 & \pm 4.12 & \pm 16.63 \\
\hline
\end{tabular}

$S D$ standard deviation, ICC intraclass correlation coefficient, SEM standard error of measurement, $C V$ coefficient of variation. 
Table 3 Relative and absolute reliability of handgrip, core, and squat and bench press strength

\begin{tabular}{|c|c|c|c|c|}
\hline \multirow[t]{2}{*}{ Parameter } & \multirow{2}{*}{$\begin{array}{l}\text { Pooled } \\
\text { mean } \pm \text { SD }\end{array}$} & \multirow{2}{*}{$\begin{array}{l}\text { Relative reliability } \\
\mathrm{ICC}_{3.1}\end{array}$} & \multicolumn{2}{|c|}{ Absolute reliability } \\
\hline & & & SEM & $\mathrm{CV}$ \\
\hline Ventral (s) & $115.0 \pm 37.4$ & 0.89 & \pm 12.66 & \pm 11.00 \\
\hline Lateral (s) & $48.6 \pm 21.0$ & 0.86 & \pm 7.90 & \pm 16.24 \\
\hline Dorsal (s) & $62.2 \pm 13.5$ & 0.78 & $\times / \div 1.11$ & $\times / \div 10.71$ \\
\hline Handgrip strength right (kg) & $50.8 \pm 10.6$ & 0.97 & \pm 1.96 & \pm 3.87 \\
\hline Handgrip strength left (kg) & $48.0 \pm 10.7$ & 0.95 & \pm 2.50 & \pm 5.22 \\
\hline 1 RM bench press (kg) & $87.3 \pm 24.0$ & 0.99 & \pm 2.48 & \pm 2.84 \\
\hline 1 RM squat (kg) & $135.5 \pm 35.9$ & 0.94 & \pm 8.7 & \pm 6.4 \\
\hline
\end{tabular}

1 RM one repetition max, SD standard deviation, ICC intraclass correlation coefficient, SEM standard error of measurement, $C V$ coefficient of variation, " \pm " error in absolute form, " $\times 1 \div$ " error as ratio, based on log transformed data.

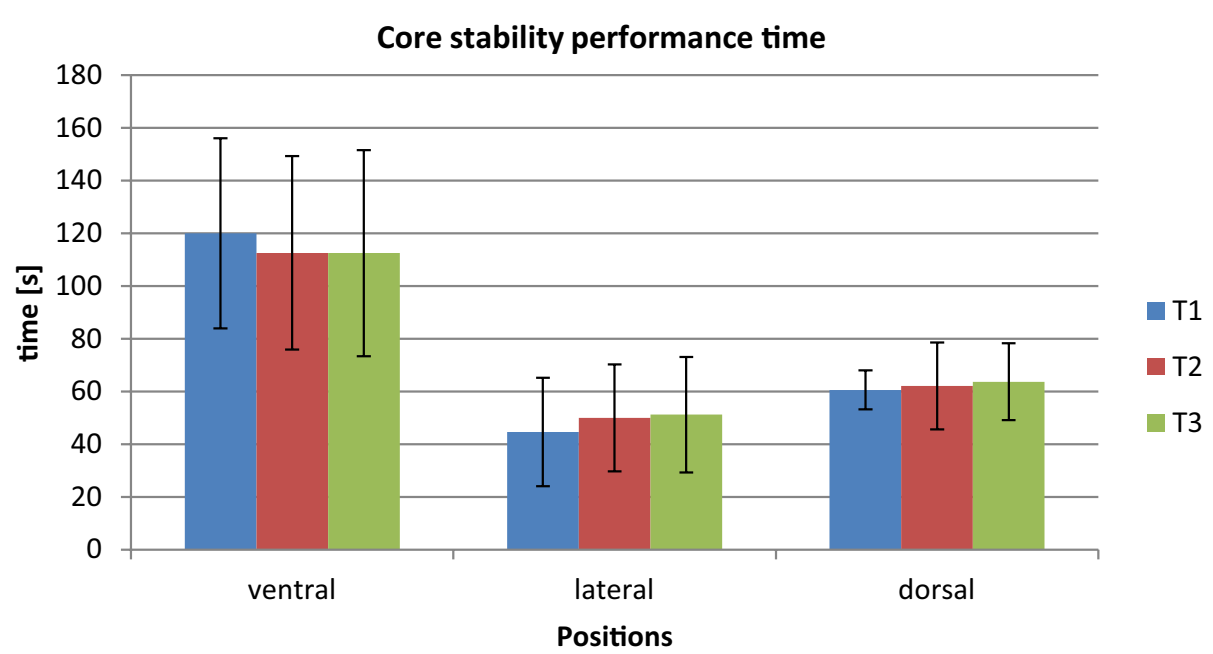

Fig. 3 Mean (+SD) core strength test performance time (s) for the ventral, lateral and dorsal position on the three test days.

\section{Muscle power}

Correlations ranged from $\mathrm{ICC}_{3.1}=0.85 ; \mathrm{CV}=21.2 \%$ for CMJ RFD, to $\mathrm{ICC}_{3.1}=0.14 ; \mathrm{CV}=14.8 \%$ for SLJ-L height. A systematic error $(F=5.09, P=0.02)$ was only identified for SLJ-R RFD (Table 5). The correlation for drop jump RSI was $\mathrm{ICC}_{3.1}=0.73$; $\mathrm{SEM}=0.15$.

\section{Aerobic capacity}

Measured (spirometry) and estimated (lactate/ ERGONIZER $\left.{ }^{\circledR}\right) \quad V \mathrm{O}_{2 \max }$ showed correlations of $\mathrm{ICC}_{3.1}$ $=0.91 ; \mathrm{SEM}=1.62 \mathrm{ml} \mathrm{kg}^{-1} \mathrm{~min}^{-1}$ and $\mathrm{ICC}_{3.1}=0.91$; $\mathrm{CV}=4.98 \%$, respectively (Table 6; Fig. 4).

\section{Discussion}

This study investigated the test-retest reliability of the current ESA AFA. It is the first time that this battery of tests has been assessed for their reliability, which is crucial for the future implementation of the AFA with ESA astronauts, to provide relevant feedback in relation to fitness performance and post-flight recovery from exposure to $\mu \mathrm{G}$.

ESA's AFA concept of performing physical fitness evaluations wherever ESA crew members are located for training or missions, requires the use of local nonportable hardware (Smith machine, treadmill, stationary gas analysis), which potentially decreases data reliability and comparability between tests performed in different locations. However, this is unavoidable until testing hardware is harmonised across all space agencies or all ESA astronauts are, without exception, assessed in one location, neither of which will happen in the near future. As such, the resulting imprecision needs to be accepted and robust assessment methods must be prioritised over sensitive or unreliable tests.

Although there is considerable variability in the data $\left(\mathrm{ICC}_{3.1}\right.$ ranging from 0.03 to 0.99$), 28$ of the 41 variables demonstrate reliability above 0.6 (Table 7), which is considered marginally acceptable for occupational field 
Table 4 Relative and absolute reliability of balance parameters

\begin{tabular}{|c|c|c|c|c|}
\hline \multirow[t]{2}{*}{ Parameter } & \multirow[t]{2}{*}{ Pooled mean \pm SD } & \multirow{2}{*}{$\begin{array}{l}\text { Relative reliability } \\
\mathrm{ICC}_{3.1}\end{array}$} & \multicolumn{2}{|c|}{ Absolute reliability } \\
\hline & & & SEM & CV \\
\hline Sway area L1 (mm²) & $22.5 \pm 12.6$ & 0.07 & \pm 12.20 & \pm 54.33 \\
\hline Sway area L2 (mm²) & $114.8 \pm 58.2$ & 0.57 & $\times / \div 1.37$ & $\times / \div 36.75$ \\
\hline Sway area L3 (mm²) & $894.2 \pm 882.0$ & 0.58 & $x / \div 1.66$ & $\times / \div 66.15$ \\
\hline Sway area L4 (mm²) & $45.4 \pm 38.3$ & 0.60 & \pm 24.24 & \pm 53.35 \\
\hline Sway area L5 (mm²) & $161.3 \pm 188.6$ & 0.59 & $\times / \div 1.71$ & $\times / \div 70.75$ \\
\hline Sway area L6 $\left(\mathrm{mm}^{2}\right)$ & $1,124.0 \pm 1,831.1$ & 0.39 & $\times / \div 1.90$ & $\times / \div 89.71$ \\
\hline COP path length L1 (mm) & $84.8 \pm 27.5$ & 0.21 & \pm 24.53 & \pm 28.94 \\
\hline COP path length L2 (mm) & $346.7 \pm 98.8$ & 0.59 & \pm 63.23 & \pm 18.24 \\
\hline COP path length L3 (mm) & $1,036.5 \pm 433.6$ & 0.63 & \pm 263.47 & \pm 25.42 \\
\hline COP path length $L 4(\mathrm{~mm})$ & $92.9 \pm 54.2$ & 0.80 & $\times / \div 1.25$ & $\times / \div 24.51$ \\
\hline COP path length $L 5(\mathrm{~mm})$ & $308.7 \pm 141.5$ & 0.61 & $\times / \div 1.26$ & $\times / \div 26.37$ \\
\hline COP path length $L 6(\mathrm{~mm})$ & $941.7 \pm 327.6$ & 0.62 & \pm 202.95 & \pm 21.55 \\
\hline Average rotation velocity L7 (\%/s) & $2.4 \pm 1.3$ & 0.54 & $\times / \div 1.43$ & $\times / \div 42.69$ \\
\hline Average rotation velocity L8 (\%/s) & $3.1 \pm 1.4$ & 0.55 & $\times / \div 1.32$ & $\times / \div 32.38$ \\
\hline Average rotation velocity $L 9(\% / s)$ & $3.3 \pm 1.4$ & 0.04 & \pm 1.38 & \pm 42.14 \\
\hline Average rotation velocity $\mathrm{L} 10(\% / \mathrm{s})$ & $3.0 \pm 1.1$ & 0.35 & \pm 0.85 & \pm 28.21 \\
\hline
\end{tabular}

SD standard deviation, ICC intraclass correlation coefficient, SEM standard error of measurement, $C V$ coefficient of variation, $L$ Level, COP centre of pressure, " \pm " error in absolute form, " $\times 1 \div$ " error as ratio, based on log transformed data.

Table 5 Relative and absolute reliability of jump parameters

\begin{tabular}{|c|c|c|c|c|}
\hline \multirow[t]{2}{*}{ Parameter } & \multirow{2}{*}{$\begin{array}{l}\text { Pooled } \\
\text { mean } \pm \text { SD }\end{array}$} & \multirow{2}{*}{$\begin{array}{l}\text { Relative } \\
\text { reliability } \\
\mathrm{ICC}_{3.1}\end{array}$} & \multicolumn{2}{|c|}{ Absolute reliability } \\
\hline & & & SEM & CV \\
\hline $\begin{array}{l}\text { Max flight height } \\
\text { CMJ (m) }\end{array}$ & $0.35 \pm 0.09$ & 0.63 & $\times / \div 1.14$ & $x / \div 14.44$ \\
\hline $\begin{array}{l}\text { Max flight height } \\
\text { SJ (m) }\end{array}$ & $0.32 \pm 0.06$ & 0.76 & $\times / \div 1.10$ & $\times / \div 9.76$ \\
\hline $\begin{array}{l}\text { Max flight height } \\
\text { SLJ-R (m) }\end{array}$ & $0.15 \pm 0.03$ & 1 & \pm 0.02 & \pm 13.09 \\
\hline $\begin{array}{l}\text { Max flight height } \\
\text { SLJ-L (m) }\end{array}$ & $0.15 \pm 0.02$ & 0.14 & $\times / \div 1.15$ & $\times / \div 14.76$ \\
\hline $\begin{array}{l}\text { Max flight height } \\
\text { DJ (m) }\end{array}$ & $0.24 \pm 0.06$ & 0.79 & \pm 0.03 & \pm 11.67 \\
\hline $\begin{array}{l}\mathrm{RFD}_{\max } \mathrm{CMJ} \\
\left(\mathrm{N} \mathrm{s}^{-1}\right)\end{array}$ & $11,553 \pm 7,275$ & 0.85 & $\times / \div 1.21$ & $\times / \div 21.18$ \\
\hline $\mathrm{RFD}_{\max } \mathrm{SJ}\left(\mathrm{N} \mathrm{s}^{-1}\right)$ & $11,018 \pm 5,192$ & 0.74 & $\times / \div 1.24$ & $\times / \div 24.25$ \\
\hline $\begin{array}{l}\mathrm{RFD}_{\max } \mathrm{SLJ}-\mathrm{R} \\
\left(\mathrm{N} \mathrm{s}^{-1}\right)\end{array}$ & $8,634 \pm 4,478$ & 0.85 & $\times / \div 1.20$ & $\times / \div 20.10$ \\
\hline $\begin{array}{l}\mathrm{RFD}_{\text {max }} \mathrm{SLJJ-L} \\
\left(\mathrm{N} \mathrm{s}^{-1}\right)\end{array}$ & $8,135 \pm 4,324$ & 0.69 & $\times / \div 1.32$ & $\times / \div 31.98$ \\
\hline $\mathrm{RFD}_{\max } \mathrm{DJ}\left(\mathrm{N} \mathrm{s}^{-1}\right)$ & $394,012 \pm 240,961$ & 0.65 & $\times / \div 1.44$ & $\times / \div 43.76$ \\
\hline $\mathrm{RSI}(\mathrm{m} / \mathrm{s})$ & $1.15 \pm 0.29$ & 0.73 & \pm 0.15 & \pm 13.03 \\
\hline
\end{tabular}

$C M J$ countermovement jump, $S J$ squat jump, $S L J-R / L$ single-leg jump right/left leg, $D J$ drop jump, $R F D$ rate of force development, $R S /$ reactive strength index, $S D$ standard deviation, ICC intraclass correlation coefficient, SEM standard error of measurement, $C V$ coefficient of variation, " \pm " error in absolute form, " $\times / \div$ " error as ratio, based on log transformed data. testing that is subject to multiple limitations and over long time periods [24]. For operational purposes, it is important to demonstrate that the elements of the fitness assessment remain at acceptable levels of reliability under different conditions. Good correlations were shown for height, body mass and percentage body fat, which were expected. Hip flexibility (Thomas Test and Sitand-Reach), muscle strength (1 RM, handgrip and core strength) and $V \mathrm{O}_{2 \max }$ (both measured and estimated) also demonstrated acceptable reliability, suggesting that, as long as sufficient standardisation is implemented, traditional assessment methods are satisfactory for physical performance measurements [1, 25-29].

Sit-and-Reach hip flexibility showed a high correlation $\left(\mathrm{ICC}_{3.1}=0.99\right)$, which is consistent with previous studies of this field-based test [25]. For longer test intervals, which are the reality for astronauts, greater differences in flexibility might occur. Thus, given its high reliability and relevance for returning astronauts-who demonstrate reduced flexibility after landing (unpublished data from ESA astronauts) - and for health in general, this finding supports maintaining this measurement in the AFA battery.

The modified Thomas Test assessing hip extension flexibility showed lower correlations compared to those reported in the literature [26-28], although are still considered acceptable. Contrary to our study, a goniometer 
Table 6 Relative and absolute reliability of maximal aerobic capacity $\left(\mathrm{VO}_{2 \max }\right)$

\begin{tabular}{lllll}
\hline Parameter & Pooled mean \pm SD & Relative reliability & Absolute reliability \\
\cline { 3 - 4 } & & ICC $_{\mathbf{3 . 1}}$ & SEM & \pm 1.62 \\
\hline $\mathrm{V}_{2 \text { max }}$ Spirometry $\left(\mathrm{ml} \mathrm{kg}^{-1} \mathrm{~min}^{-1}\right)^{\mathrm{a}}$ & $54.7 \pm 5.4$ & 0.91 & \pm 2.97 \\
$V \mathrm{O}_{2 \text { max }}$ lactate $\left(\mathrm{ml} \mathrm{kg} \mathrm{min}^{-1}\right)^{\mathrm{b}}$ & $49.3 \pm 7.4$ & 0.91 & $\times / \div 1.05$ & $\times / \div 4.98$ \\
\hline
\end{tabular}

SD standard deviation, ICC intraclass correlation, SEM standard error of measurement, CV coefficient of variation coefficient, " \pm " error in absolute form, " $\times / \div$ " error as ratio, based on log transformed data.

a Measured directly using spirometry.

b Estimated from lactate values using ERGONIZER ${ }^{\circledR}$ software.

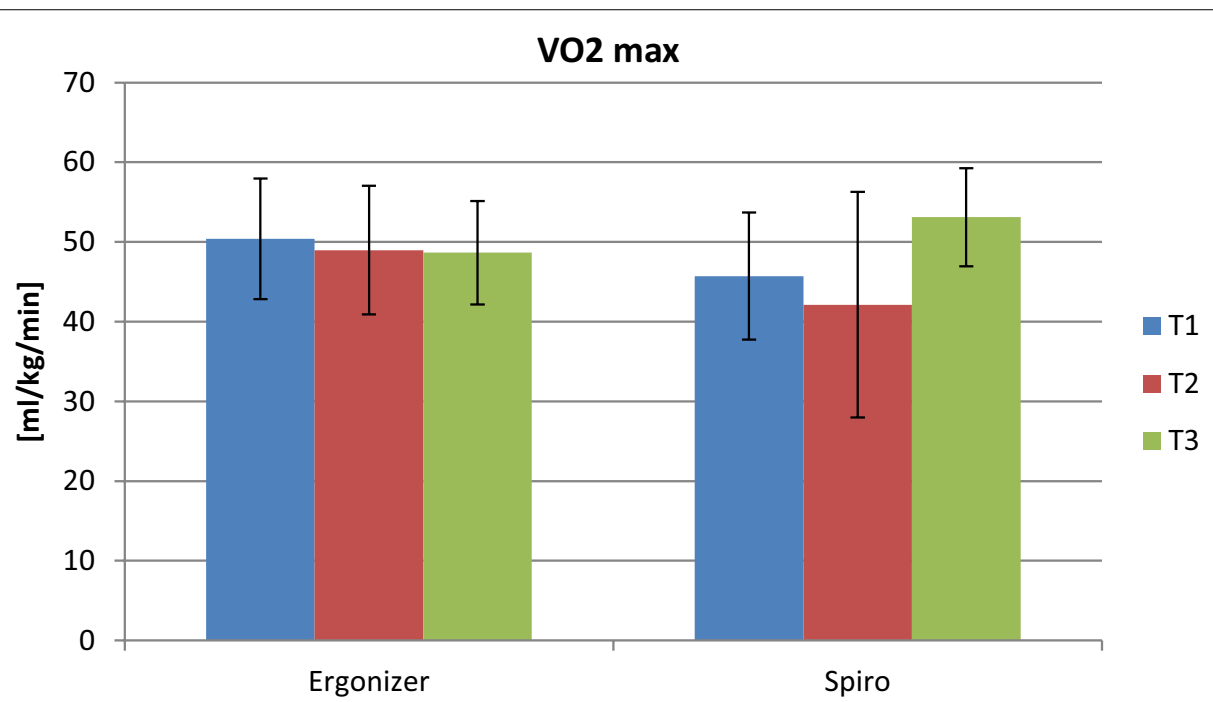

Fig. 4 Mean (+SD) measured (via spirometry) and estimated (from blood lactate samples and using the ERGONIZER ${ }^{\circledR}$ software) aerobic capacity on the three test days.

was used in these studies, which may provide better precision compared with an inclinometer, which we chose for reasons of time efficiency. To obtain reliable results, it appears advisable for measurements to be implemented by a well-trained examiner [30], and the experimenter in this study (and ESA staff members who conduct the AFA) was trained to perform the test. As such, to improve the quality of this test, the identification and use of a timeefficient goniometer setup should be prioritised.

The high reliability seen for muscular strength assessments are encouraging, and reflect the careful manner in which they were implemented. Measurement bias was detected with the $F$ test for the estimate of squat 1 RM which might reflect a training effect; although all participants had experience with weight lifting, not all of them performed weekly resistance training, and thus potentially "profited" from this additional training stimulus. A stable form when performing a maximum effort squat and bench press evaluation is required for providing consistent values [17, 31]. The simple, multiple repetition estimation method used in the present study appears appropriate for application with astronauts, who, in preparation for and during space missions, perform daily exercises including the squat and bench press, by default, develop sufficient technical skills and are considered sufficiently experienced weight lifters to produce reliable data.

Handgrip strength appears a fast, simple and reliable measure, and thus warrants being maintained in the AFA test battery. For core strength, based on the present findings, one might consider removing the dorsal and lateral position tests from the AFA, and only keeping the ventral position, although all three tests showed acceptable reliability. Although it was not assessed in this study, a trend for decreased performance was visible in the lateral and dorsal position data. This might be related to shoulder or hamstring fatigue occurring prior to onset of trunk muscle fatigue and possibly as a result of the order of testing (ventral always first). Reducing the core test to a single position would enhance time efficiency and likely reduce fatigue effects, so future work in this area should focus on identifying possible 
Table 7 All parameters ranked (highest to lowest) by relative $\left(\mathrm{ICC}_{3.1}\right)$ reliability

\begin{tabular}{|c|c|c|c|}
\hline Parameter & $\mathrm{ICC}_{3.1}$ & SEM & $\mathrm{CV}$ \\
\hline Height (cm) & 0.99 & \pm 0.23 & \pm 0.13 \\
\hline Body mass (kg) & 0.99 & \pm 0.73 & \pm 0.95 \\
\hline 1 RM bench press $(\mathrm{kg})$ & 0.99 & \pm 2.48 & \pm 2.84 \\
\hline Sit-and-Reach (cm) & 0.99 & \pm 1.20 & \pm 4.41 \\
\hline Handgrip strength R (kg) & 0.97 & \pm 1.96 & \pm 3.87 \\
\hline Handgrip strength L (kg) & 0.95 & \pm 2.50 & \pm 5.22 \\
\hline 1RM Squat (kg) & 0.94 & \pm 8.67 & \pm 6.4 \\
\hline$V O_{2 \max }\left(\right.$ Spirometry) $\left(\mathrm{ml} \mathrm{kg}^{-1} \mathrm{~min}^{-1}\right)^{\mathrm{a}}$ & 0.91 & \pm 1.62 & \pm 2.97 \\
\hline$V O_{2 \max }($ lactate $)\left(\mathrm{ml} \mathrm{kg}^{-1} \mathrm{~min}^{-1}\right)^{\mathrm{b}}$ & 0.91 & $\times / \div 1.05$ & $\times / \div 4.98$ \\
\hline Body fat (\%) & 0.89 & \pm 1.80 & \pm 15.81 \\
\hline Core strength ventral (s) & 0.89 & \pm 12.66 & \pm 11.00 \\
\hline Core strength lateral (s) & 0.86 & \pm 7.90 & \pm 16.24 \\
\hline Thomas test right $\left({ }^{\circ}\right)$ & 0.85 & \pm 2.69 & \pm 11.63 \\
\hline $\operatorname{RFD} C M J\left(\mathrm{~N} \mathrm{~s}^{-1}\right)$ & 0.85 & $\times / \div 1.21$ & $\times / \div 21.18$ \\
\hline RFD SLJ-R $\left(\mathrm{N} \mathrm{s}^{-1}\right)$ & 0.85 & \pm 1.20 & \pm 20.10 \\
\hline Path length L4 (mm) & 0.80 & $\times / \div 1.25$ & $\times / \div 24.51$ \\
\hline Max height DJ (m) & 0.79 & \pm 0.03 & \pm 11.67 \\
\hline Core strength dorsal (s) & 0.78 & \pm 1.11 & \pm 10.71 \\
\hline Max height SJ (m) & 0.76 & $\times / \div 1.10$ & $\times / \div 9.76$ \\
\hline RFD SJ $\left(\mathrm{N} \mathrm{s}^{-1}\right)$ & 0.74 & $\times / \div 1.24$ & $\times / \div 24.25$ \\
\hline $\mathrm{RSI}\left(\mathrm{m} \mathrm{s}^{-1}\right)$ & 0.73 & \pm 0.15 & \pm 13.03 \\
\hline RFD SLJ-L $\left(\mathrm{N} \mathrm{S}^{-1}\right)$ & 0.69 & $\times / \div 1.32$ & $\times / \div 31.98$ \\
\hline RFD DJ $\left(\mathrm{N} \mathrm{s}^{-1}\right)$ & 0.65 & $\times / \div 1.44$ & $\times / \div 43.76$ \\
\hline Path length $\mathrm{L} 3(\mathrm{~mm})$ & 0.63 & \pm 263.47 & 25.42 \\
\hline max height CMJ (m) & 0.63 & $\times / \div 1.14$ & $\times / \div 14.44$ \\
\hline Thomas test left $\left({ }^{\circ}\right)$ & 0.63 & \pm 4.12 & \pm 16.63 \\
\hline Path length $L 6(\mathrm{~mm})$ & 0.62 & \pm 202.95 & \pm 21.55 \\
\hline Path length L5 (mm) & 0.61 & $\times / \div 1.26$ & $\times / \div 26.37$ \\
\hline Sway area L4 (mm²) & 0.6 & \pm 24.24 & \pm 53.35 \\
\hline Sway area L5 ( $\left.\mathrm{mm}^{2}\right)$ & 0.59 & $\times / \div 1.71$ & $\times / \div 70.75$ \\
\hline Path length L2 (mm) & 0.59 & \pm 63.23 & \pm 18.24 \\
\hline Sway area L3 (mm²) & 0.58 & $\times / \div 1.66$ & $\times / \div 66.15$ \\
\hline Sway area L2 (mm²) & 0.57 & $\times / \div 1.37$ & $\times / \div 36.75$ \\
\hline Average rotation speed L8 (\%/s) & 0.55 & $\times / \div 1.32$ & $\times / \div 32.38$ \\
\hline Average rotation speed L7 ( $\%$ s) & 0.54 & $\times / \div 1.43$ & $\times / \div 42.69$ \\
\hline Max height SLJ-R (m) & 0.51 & \pm 0.02 & \pm 13.09 \\
\hline Sway area L6 (mm²) & 0.39 & $\times / \div 1.90$ & $\times / \div 89.71$ \\
\hline Average rotation speed L10 (\%/s) & 0.35 & \pm 0.85 & \pm 28.21 \\
\hline Path length L1 (mm) & 0.21 & \pm 24.53 & \pm 28.94 \\
\hline max height SLJ-L (m) & 0.14 & $\times / \div 1.14$ & $\times / \div 14.76$ \\
\hline Sway area L1 (mm²) & 0.07 & $\times / \div 12.20$ & $\times / \div 54.33$ \\
\hline Average rotation speed $\mathrm{L} 9(\% / \mathrm{s})$ & 0.04 & \pm 1.38 & \pm 42.14 \\
\hline
\end{tabular}

" \pm " absolute error based on raw data, $C M J$ countermovement jump, SJ squat jump, $S L-R / L$ single-leg jump right/left leg, $D J$ drop jump, RFD rate of force development, $R S /$ reactive strength index, $L$ Level, $S D$ standard deviation, ICC intraclass correlation coefficient, SEM standard error of measurement, $C V$ coefficient of variation, " \pm " error in absolute form, " $\times / \div$ " error as ratio, based on log transformed data.

a Measured directly using spirometry.

${ }^{\text {b }}$ Estimated from lactate values using ERGONIZER ${ }^{\circledR}$ software. carryover effects with multiple tests and which of the three tests best reflects overall core muscle function. If only one test were to be selected, the ventral position might be preferable, as it is more stable and potentially less fatiguing that the others, as it places considerable load on other muscle groups (e.g. shoulders, hamstrings).

Balance and jump tests both show marked variation in measured values between test days, with $\mathrm{ICC}_{3.1}$ ranging from 0.14 to 0.85 for jumps and 0.04 to 0.80 for balance, whereas the highest value was shown for the countermovement jump RFD and for balance COP path length L4 (both legs, head tilted back), respectively. Although very reliable, the measurement of static head tilt back (L4) balance capability might not be the strongest parameter for assessing the quality of sensory organisation [32]. The test may still show high reliability without being associated with high validity, which needs to be further investigated.

For balance tests, reliability above $\mathrm{ICC}_{3.1}=0.6$ was found for COP path length on Level 3-6 on the pressure plate $\left(\mathrm{ICC}_{3.1}=0.61-0.80\right)$, thus path length appeared the more reliable parameter compared to COP sway area $\left(\mathrm{ICC}_{3.1}=0.06-0.60\right)$, which is consistent with the literature $[33,34]$. The lowest reliability was seen for balance tasks on the balance board (rotation velocity L8, L7, L10, L9) $\left(\mathrm{ICC}_{3.1}=0.04-0.55\right)$, although both sway area $\left(\mathrm{ICC}_{3.1}=0.07\right)$ and path length $\left(\mathrm{ICC}_{3.1}=0.21\right)$ at $\mathrm{L} 1$ on the pressure platform also demonstrated low values. Given the relative simplicity of the task (both legs, eyes closed), this was surprising, but, being the first balance task each time, it could be related to an initial familiarisation effect (comparable to a warm-up). A repetition of the first task might have removed/reduced this effect, although such a strategy has not been reported in other studies, and subjects in the present study did complete the test during their familiarisation session prior to the study itself.

Ten out of 16 balance parameters displayed ICCs below 0.6 , which questions their suitability for inclusion in the AFA. The high random error observed in the present study, despite standardisation of positioning, time and abort criteria, appears a common problem with balance assessments [35], and the influence factors have not been fully identified [34, 36]. Environmental interferences, such as noise or visual stimuli, possibly distracting the subject or day to day changes $[1,37]$, may affect results, but are difficult to quantify and sometimes unavoidable in field testing. Other studies [35, 36, 38-40] report that balance performance and the reliability of results also vary with trial duration. The task duration of $15 \mathrm{~s}$ in the present study might, therefore, be too short and could be extended [24] and, for time efficiency, the application of a 
smaller number of longer duration tasks and/or more trials with each task might be a better approach.

A high sampling rate of $100 \mathrm{~Hz}$ increases the sensitivity of pressure plate measurements and may contribute to higher variance as more data points are tracked [34]. A high sampling rate alone, however, does not seem to increase variance, as shown by Muehlbauer et al. [36], who, applying $400 \mathrm{~Hz}$, obtained good reliability for intra- and inter-session sway area (ICC $>0.77$ and 0.87 ) with male participants [36]. Thus, modification of sampling rate may be one, but likely not the only solution for improving reliability in our test setup. A reduction of sampling rate would not affect assessment time, but potentially measurement precision. Sampling rate and test sensitivity may, however, be unrelated, and to obtain certainty, the effect of modifying this parameter would need to be demonstrated with the given test protocol.

The implementation of a balance assessment on an instrumented balance board may well be a novel strategy and a review of the literature revealed no published studies using such a device. The test was included in the battery of balance tests to provide a level of difficulty sufficient to challenge well-trained, younger astronauts in their annual assessments. However, given the low reliability seen in the data, it is possible that the test was simply too difficult to achieve consistent performance. As such, as the primary goal of the AFA is to detect changes in ESA astronaut performance, the removal of the balance board tests should, therefore, be considered.

In the context of astronaut testing, the variability or measurement error should not mask real adaptation effects seen after spaceflight to be a meaningful test. At this stage we cannot confirm that this is the case here, and thus either modification (increase of duration and/ or number of trials, modification of sampling rate) or elimination of some balance tasks (e.g. on balance board) should be considered.

Microgravity is known to impact neuro-muscular control mechanisms [10-12], which may affect astronauts' post-flight muscle power development. The double-legged jump tests showed good reliability as has been reported previously [41, 42], and thus should produce meaningful post-flight data. A marked variation in jump values was observed, showing low reliability for SLJ max height for SLJ-L $\left(\mathrm{ICC}_{3.1}=0.14\right)$, and SLJ-R $\left(\mathrm{ICC}_{3.1}=0.51\right)$, although all other jump parameters showed ICCs higher than 0.6. For small samples like ours, the mean of all trials could be used instead of the single best effort, to smoothen samples with artefacts. Low reliability in the SLJ may be also related to the technical difficulty of performance, which requires considerable balance. Jump performance variation decreases with increased jumping experience [41, 43, 44], although large variation in vertical jump mechanical variables measured on force platforms occur even in highly trained athletes, and may thus affect reliability [41], and this could be an even bigger factor in astronauts, who are not professional athletes and cover a wide age range (27-60 years). With low reliability in our test population and the potential for even greater variation due to post-flight balance issues experienced by astronauts (and thus also safety concerns), our data suggest that the SLJ tests should be considered for removal from the AFA test battery.

Spirometry and lactate assessments (either alone or in combination) during treadmill running are established measurements for fitness evaluation [45-48]. An estimate and direct measurement of $V_{2}$ max from lactate measurements and spirometry produced high and comparable reliability, and thus appear appropriate for operational use with ESA astronauts. Based on the good reliability results with the ERGONIZER ${ }^{\circledR}$ software, this suggests that it could be used independently from spirometry on the occasions when the spirometry equipment is not available for the AFA to make a direct measurement. We did not assess redundancy between both methods, although this would be useful and should be considered for future evaluation.

There are a number of limitations to this study. We acknowledge that the number of subjects is low, which is a result of the currently limited capacity of ESA staff members to take time away from their operational support roles to perform research activities, as well as to use the test equipment-that is required for performing the AFA with ESA crew members-for non-operational purposes. This limitation was mitigated through the implementation of thorough statistics, including screening data for normality and homoscedasticity, to adequately assess measurement bias and reliability. However, the observed measures of reliability should be treated only as provisional and final decisions as to whether tests should be used in the AFA, modified or removed, should not be taken until each test has been investigated in the respective occupational group, with a larger sample size and in the operational context.

The complexity and length of the test battery and the study conditions may lead to limitations. The pre-set time limitations, hardware portability and the amount of tests to be covered within a 2 -h time slot per subject reduce the ability to perform higher standard laboratory assessments and multiple repetitions to increase reliability.

No inter-equipment reliability assessment was performed as part of this study. Currently, the AFA has been conducted at three different sites: the European Astronaut Centre (EAC) in Cologne, Germany, NASA Johnson Space Centre (JSC) in Houston, USA and Gagarin Cosmonaut Training Centre (GCTC) ("Star City"), near 
Moscow in Russia. European Astronaut Centre equipment is transported for AFAs in Russia, so inter-equipment issues are not an issue in these instances, but they could be when the AFA is conducted at JSC, where a duplicate set of equipment is stored and used. However, as all of the hardware/software used is commercial-offthe-shelf, the variation in construction and performance of different units is likely to be small and thus the influence of inter-equipment variability minimal. Furthermore, although for any individual astronaut, AFAs are set up and conducted in their entirety by one person, three people are qualified to administer the AFA and, it is possible that tests may not be conducted by the same person (e.g. due to illness). As such, inter-observer variation might be an issue for the AFA. We did not address this issue in the present study, but it will be the subject of future investigations.

Although learning and fatigue effects were minimised through weekly sessions, allowing for sufficient recovery and reducing short-time memory, they may have still occurred (e.g. trends in squats or handgrip strength) in the analysed data set. Additional familiarisation sessions or trials prior to measurement could have mitigated this effect, but increased time demands. The testing environment and procedures were standardised to the maximal possible extent with the intention to minimise systematic bias, to identify the random error of measurement, and to ultimately allow a distinction from real performance changes. However, not all external sources of noise or distraction could be fully eliminated nor their effect on results clearly quantified in the data.

\section{Conclusions}

Measurement of height, body mass and percentage body fat, hip flexion/extension, muscular strength (handgrip, core strength and repetition maximum for squat and bench press), double-legged jumps and balance parameters on the pressure plate appear, with minor adjustments enhancing precision, to be adequate for operational implementation of the AFA in the "field test" conditions required for human space flight. Balance tasks implemented on the spherical balance board and single-leg jumps did not demonstrate sufficient reliability, revealing high random error, which could potentially mask effects of $\mu \mathrm{G}$ on astronauts returning from missions. Given practical considerations of operational implementation, mainly those of time constraints, safety aspects, high data complexity and low reliability, and in view of yet undetermined occupational relevance, a comprehensive redesign considering shortening and simplification of the balance protocol is recommended. For similar reasons, the single-leg jumps should be considered for elimination from the battery. High standardisation of procedures should be targeted to mitigate the impact of external factors. Overall the other AFA elements showed acceptable reliability, requiring minor corrections, for continued operational use and further development in the given conditions of space medicine applied in European space flight.

\section{Abbreviations}

AFA: Astronaut Fitness Assessment; ANOVA: analysis of variance; CMJ: countermovement jump; COP: centre of pressure; CV: coefficient of variation; DJ: drop jump; EAC: European Astronaut Centre; ESA: European Space Agency; GRF: ground reaction force; ICC: intraclass correlation coefficient; ISS: International Space Station; L: Level; RFD: rate of force development; RM: repetition maximum; RSI: reactive strength index; SEM: standard error of measurement; SJ: squat jump; SLJ-R/L: single-leg jump right/left leg; $V_{O_{2 m a x}}$ maximal oxygen uptake; $\mu \mathrm{G}$ : microgravity.

\section{Authors' contributions}

NP designed, planned and carried out the study, including statistical data analysis, data interpretation and writing of the manuscript. LT participated in planning and carrying out the study, statistical data analysis and manuscript preparation. LPS helped in the data analysis and interpretation, participated in the drafting and revision of the manuscript. VD participated in the design, coordination and formal approval of the study, revised the manuscript and gave final approval for publication. JM conceived of the study, participated in the design and coordination and approval process, provided access to test facilities and critically revised and approved the manuscript for publication. All authors read and approved the final manuscript.

\section{Author details}

${ }^{1}$ Wyle GmbH, Cologne, Germany. ${ }^{2}$ Space Medicine Office (HSO-AM), European Astronaut Centre Department (ESA), Directorate of Human Spaceflight and Operations (D/HSO), European Space Agency, Geb. 12, Linder Höhe, PO Box 906096, 51147 Cologne, Germany. ${ }^{3}$ Institute of Physiology and Anatomy, German Sport University Cologne (DSHS), Am Sportpark Muengersdorf 6, 50955 Cologne, Germany. ${ }^{4}$ Universities Space Research Association, NASA Johnson Space Center, B261, SK3, Houston, TX 77058, USA. ${ }^{5}$ Institute of Training Science and Sport Informatics, German Sport University Cologne (DSHS), Am Sportpark Muengersdorf 6, 50933 Cologne, Germany.

\section{Acknowledgements}

Jonathan Scott for substantial support in writing and structuring and the manuscript. Tobias Weber and Andre Rosenberger for critically reviewing the manuscript. Patrick Jaekel for providing operational procedural support during implementation of the study. Members of the DSHS Institute of Training Science and Sport Informatics for supporting the study activities implemented at their laboratory. Casey Pruett, Managing Director of Wyle Laboratories GmbH, for managerial and moral support.

\section{Compliance with ethical guidelines}

\section{Competing interests}

The authors declare that they have no competing interests.

Received: 26 May 2015 Accepted: 31 July 2015

Published online: 14 August 2015

\section{References}

1. Deakin JM, Pelot R, Eng P, Smith JT, Weber CL (2000) Development and validation of canadian forces minimum physical fitness standard (MPFS 2000). Ergonomics Research Group Queen's University, Kingston

2. Serra C, Rodriguez MC, Delclos GL, Plana M, Gomez Lopez LI, Benavides FG (2007) Criteria and methods used for the assessment of fitness for work: a systematic review. Occup Environ Med 64(5):304-312. doi:10.1136/oem.2006.029397 
3. Trappe S, Costill D, Gallagher P, Creer A, Peters JR, Evans H et al (2009) Exercise in space: human skeletal muscle after 6 months aboard the International Space Station. J Appl Physiol (1985) 106(4):1159-1168. doi:10.1152/japplphysiol.91578.2008

4. Gopalakrishnan R, Genc KO, Rice AJ, Lee SM, Evans HJ, Maender CC et al (2010) Muscle volume, strength, endurance, and exercise loads during 6-month missions in space. Aviat Space Environ Med 81(2):91-102

5. Shackelford LC, LeBlanc AD, Driscoll TB, Evans HJ, Rianon NJ, Smith SM et al (2004) Resistance exercise as a countermeasure to disuseinduced bone loss. J Appl Physiol (1985) 97(1):119-129. doi:10.1152/ japplphysiol.00741.2003

6. Smith SM, Heer MA, Shackelford LC, Sibonga JD, Ploutz-Snyder L, Zwart SR (2012) Benefits for bone from resistance exercise and nutrition in longduration spaceflight: evidence from biochemistry and densitometry. J Bone Miner Res 27(9):1896-1906. doi:10.1002/jbmr.1647

7. Moore AD, Lee SMC, Stenger MB, Platts SH (2010) Cardiovascular exercise in the U.S. space program: past, present and future. Acta Astronaut 66(7-8):974-988

8. Convertino V, Sandler H (1995) Exercise countermeasures for spaceflight. Acta Astronaut 35(4-5):253-270

9. McPhee JC, Charles JB (2009) Human health and performance risk at space exploration missions. Evidence reviewed by the NASA Human Research Program. NASA SP-2009-3405

10. Wood SJ, Loehr JA, Guilliams ME (2011) Sensorimotor reconditioning during and after spaceflight. NeuroRehabilitation 29(2):185-195. doi:10.3233/ NRE-2011-0694

11. Paloski WH, Reschke MF, Black FO, Doxey DD, Harm DL (1992) Recovery of postural equilibrium control following spaceflight. Ann NY Acad Sci 656:747-754

12. Reschke MF, Bloomberg JJ, Harm DL, Paloski WH, Layne C, McDonald V (1998) Posture, locomotion, spatial orientation, and motion sickness as a function of space flight. Brain Res Brain Res Rev 28(1-2):102-117

13. Medical Evaluation Documents (MED) Volume A (2014) Medical standards for ISS crew members. Rev 3.3 SSP 50667

14. Harvey DG (1998) Assessment of the flexibility of elite athletes using the modified Thomas test. Br J Sports Med 32:68-70

15. Tschopp M (2003) Manual Leistungsdiagnostik Kraft. Swiss Olympic Medical Centers, Magglingen

16. Brzycki M (1993) Strength testing: predicting a one-rep max from reps to fatigue. J Phys Educ Recreat Dance 46(1):88-90

17. Niewiadomski W, Laskowska D, Gasiorowska A, Cybulski G, Strasz A, Langfort J (2008) Determination and prediction of one repetition maximum (1 RM): safety considerations. J Hum Kinet 19:109-120

18. Verdonck A, Wilke C (1998) Screeningverfahren. In: Froboese I, Nellessen $G$ (eds) Training in der Therapie: Grundlagen und Praxis. Ullenstein Medical, Wiesbaden, pp 116-128

19. V/Cl (1999) Kistler Force Plate Formulae. http://isbweb.org/software/ movanal/vaughan/kistler.pdf. Accessed 20 May 2015

20. Bruce RA, Cooper MN, Gey GO, Fisher LD, Peterson DR (1973) Variations in responses to maximal exercise in health and in cardiovascular disease. Angiology 24(11):691-702

21. Atkinson G, Nevill AM (1998) Statistical methods for assessing measurement error (reliability) in variables relevant to sports medicine. Sports Med 26(4):217-238

22. Hopkins WG (2000) Measures of reliability in sports medicine and science. Sports Med 30(1):1-15

23. Weir JP (2005) Quantifying test-retest reliability using the intraclass correlation coefficient and the SEM. J Strength Cond Res 19(1):231-240. doi:10.1519/15184.1

24. Burnstein BDS, Russel J, Shrier I (2011) Reliability of fitness tests using methods and time periods common in sport and occupational management. J Athl Train 46(5):505-513

25. Atamaz F, Ozcaldiran B, Ozdedeli S, Capaci K, Durmaz B (2011) Interobserver and intraobserver reliability in lower-limb flexibility measurements. J Sports Med Phys Fit 51(4):689-694

26. Dennis RJ, Finch CF, Elliott BC, Farhart PJ (2008) The reliability of musculoskeletal screening tests used in cricket. Phys Ther Sport 9(1):25-33. doi:10.1016/j.ptsp.2007.09.004

27. Glanzman AM, Swenson AE, Kim H (2008) Intrarater range of motion reliability in cerebral palsy: a comparison of assessment methods. Pediatr Phys Ther 20(4):369-372. doi:10.1097/PEP.0b013e31818b7994
28. Gabbe BJ, Bennell KL, Wajswelner H, Finch CF (2004) Reliability of common lower extremity musculoskeletal screening tests. Phys Ther Sport 5:70-90

29. Espana-Romero V, Ortega FB, Vicente-Rodriguez G, Artero EG, Rey JP, Ruiz JR (2010) Elbow position affects handgrip strength in adolescents: validity and reliability of Jamar, DynEx, and TKK dynamometers. J Strength Cond Res 24(1):272-277. doi:10.1519/JSC.0b013e3181b296a5

30. Bartlett MD, Wolf LS, Shurtleff DB, Stahell LT (1985) Hip flexion contractures: a comparison of measurement methods. Arch Phys Med Rehabil 66(9):620-622

31. Ritti-Dias RM, Avelar A, Salvador EP, Cyrino ES (2011) Influence of previous experience on resistance training on reliability of one-repetition maximum test. J Strength Cond Res 25(5):1418-1422. doi:10.1519/ JSC.0b013e3181d67c4b

32. Jain V, Wood SJ, Feiveson AH, Black FO, Paloski WH (2010) Diagnostic accuracy of dynamic posturography testing after short-duration spaceflight. Aviat Space Environ Med 81(7):625-631

33. Maribo T, Stengaard-Pedersen K, Jensen LD, Andersen NT, SchiottzChristensen B (2011) Postural balance in low back pain patients: intra-session reliability of center of pressure on a portable force platform and of the one leg stand test. Gait Posture 34(2):213-217. doi:10.1016/j. gaitpost.2011.04.014

34. Raymakers JA, Samson MM, Verhaar HJ (2005) The assessment of body sway and the choice of the stability parameter(s). Gait Posture 21(1):4858. doi:10.1016/j.gaitpost.2003.11.006

35. Ruhe A, Fejer R, Walker B (2010) The test-retest reliability of centre of pressure measures in bipedal static task conditions - a systematic review of the literature. Gait Posture 32(4):436-445. doi:10.1016/j. gaitpost.2010.09.012

36. Muehlbauer T, Roth R, Mueller S, Granacher U (2011) Intra and intersession reliability of balance measures during one-leg standing in young adults. J Strength Cond Res 25(8):2228-2234. doi:10.1519/ JSC.0b013e3181fb393b

37. Moghadam M, Ashayeri H, Salavati M, Sarafzadeh J, Taghipoor KD, Saeedi A et al (2011) Reliability of center of pressure measures of postural stability in healthy older adults: effects of postural task difficulty and cognitive load. Gait Posture 33(4):651-655. doi:10.1016/j.gaitpost.2011.02.016

38. Doyle TL, Newton RU, Burnett AF (2005) Reliability of traditional and fractal dimension measures of quiet stance center of pressure in young, healthy people. Arch Phys Med Rehabil 86(10):2034-2040. doi:10.1016/j. apmr.2005.05.014

39. Le Clair K, Roach C (1996) Postural stability measures: what to measure and for how long. Clin Biomech 11:176-178

40. Lin D, Seol H, Nussbaum MA, Madigan ML (2008) Reliability of COPbased postural sway measures and age-related differences. Gait Posture 28(2):337-342. doi:10.1016/j.gaitpost.2008.01.005

41. Moir GL, Garcia A, Dwyer GB (2009) Intersession reliability of kinematic and kinetic variables during vertical jumps in men and women. Int Sports Physiol Perform 4(3):317-330

42. Ditroilo M, Forte R, McKeown D, Boreham C, De Vito G (2011) Intra- and inter-session reliability of vertical jump performance in healthy middleaged and older men and women. J Sports Sci 29(15):1675-1682. doi:10.1 080/02640414.2011.614270

43. Makaruk H, Czaplicki A, Sacewicz T, Sadowski J (2014) The effects of single versus repeated plyometrics on landing biomechanics and jumping performance in men. Biol Sport. 31(1):9-14. doi:10.5604/20831862.1083273

44. Kurz G, Lang D, Richter A, Schwameder H (eds) (2009) Reliability of drop jump variations in performance diagnostics. In: ISBS — conference proceedings archive

45. Pfitzinger P, Freedson PS (1998) The reliability of lactate measurements during exercise. Int J Sports Med 19(5):349-357. doi:10.105 5/s-2007-971929

46. Pivarnik JM, Dwyer MC, Lauderdale MA (1996) The reliability of aerobic capacity $\left(\mathrm{VO}_{2 \max }\right)$ testing in adolescent girls. Res Q Exerc Sport 67(3):345348. doi:10.1080/02701367.1996.10607962

47. Tanner RK, Fuller KL, Ross ML (2010) Evaluation of three portable blood lactate analysers: Lactate Pro, Lactate Scout And Lactate Plus. Eur J Appl Physiol 109(3):551-559. doi:10.1007/s00421-010-1379-9

48. Vickers RRJ (2003) Measurement error in maximal oxygen uptake tests. Naval Health Research Center, San Diego. Report No.: 04-03 